\title{
Power Efficient Dynamic Source Routing Protocol
}

\author{
Salonee Mishra ${ }^{1}$ and Binod Kumar Pattanayak ${ }^{2}$ \\ Department of Computer Science and Engineering, Institute of Technical Education \\ and Research, Siksha 'O' Anusandhan University, Khandagiri Square, Bhubaneswar, \\ Odisha, India \\ Email: ${ }^{1}$ salonee.mishra2@gmail.com, ${ }^{2}$ bkp_iter@yahoo.co.in \\ Abstract
}

\begin{abstract}
Power consumption in wireless ad hoc networks is an important design concern in the modern network research scenario. It is a concern because witeless nodes run with the help of battery and it has a limited life time period If the poDer fallure of mobile node occurs it affects not only its ability to forward packets to destimation but alsolts ability to forward packets on behalf of others and hence overall network lifetime. Moreover, it is very difficult to replace or recharge a mobile node powered by batteries so in order to maximize the life time of nodes routing of traffic should be in such a way that power consumption is minimized. Hence, every effort is to be channeled towards reducing power. More precisely, network lifetime is a key design metric in MANETS. Our Mandontribution in this thesis is Power Efficient Dynamic Source Routing (PEDSR) protocol satisfying less power consumption from the viewpoints of nodes and network. To achieve our goal, first, we studied DSR protocol using performance and power aware metrics. Modifications are done on the Dynamic Source Routing (DSR) protocol by aking into consideration the outcomes of the pre-simulation, the existing feature of DSR for implementation of the design, and the previous research works done on DSR routing protocol by many researchers. Subsequently, we studied the performance evaluation of ourproposition. The simulation results show that the power aware routing protocol, PEDSR outperforms the original DSR protocol in majority of the scenarios and evaluation metrics.
\end{abstract}

Keywords: Power Consumption, Network lifetime, DSR (Dynamic source routing), PEDSR (Power Efficient Dyramic Source Routing)

\section{Introduction}

\subsection{Mobile ad-hoc Networks}

Amobile ad hoc network (MANET) [1] is an autonomous, self-configuring and infrastucture less network of mobile devices connected by wireless. Ad hoc is being derived from Latin which means "for this purpose". Manet is an infrastructure-less network since they do not have any fixed infrastructure or base station to carry out their operation. Each device in a MANET is free to move independently and randomly in any direction and hence change its links to other devices frequently. Each must forward packet traffic unrelated to its own use, and therefore be a router. In general, routes between mobile nodes in an ad hoc network may include multi hops for that reason such networks are also called as multi-hop wireless ad hoc networks. In Figure 1: MANET each device is equipped to continuously maintain the information required to properly route traffic. Such networks may operate by themselves or may be connected to the large internet. Mobile nodes self-organize to form a network over 
radio links. MANETs are a kind of Wireless ad hoc network that usually has a routable networking environment on top of a Link Layer ad hoc network.

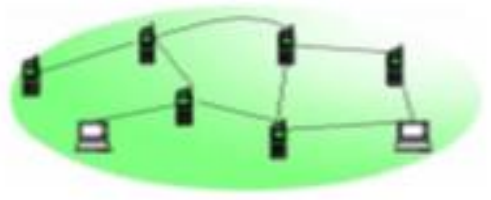

Figure 1. Mobile Ad Hoc Network

Applications of Mobile ad hoc networks include Tactical networks which provide services as military communication and operations, automated battlefields. Emergency services includes Disaster recovery, Search and rescue operations, Policing and fire fighting, Replacement of fixed infrastructure in case of environmental disasters, other applications includes Commercial and civilian which provides services as E-commerce, dynamic database access, inter-vehicle networks. Education includes Universities and campus settings, Virtual classrooms, Home and enterprise services includes Home/office wireless networking, Conferences, Personal area networks (PAN), Personal newworks (PN) etc. Context aware services includes services such as callforwarding, mobile workspace, location specific services, time dependent services

\subsection{Characteristics and Avantages of MANET}

MANET is having the characteristics of Wireless network in general, and additional characteristics that are specific to the Ad Hoe Networking:

1) WIRELESS: Nodes communicate wirelessly and share the same media (radio, infrared, etc.).

2) Ad-hoe-based: A mobile dd hoc network is a temporary network formed dynamically in an arbitrary manner by a collection of nodes as need arises.

3) AUTONOMOUS and infrastructure-less: The existing wireless infrastructure is expensive and mconvenient to use. Ad-hoc networks can be classified into three categories based on applications; Mobile Ad-hoc Networks (MANETs), Wireless Mesh Networks (WMNs)[2] and Wireless Sensor Networks (WSN)[3]. MANET does not defend on any established infrastructure or centralized administration. In infrastructure less wireless network, during communication only the mobile nodes moye without any base station and all the nodes in the network act as routers. Each

node operates in distributed peer-to-peer mode, acts as an independent router, and generates independent data.

4) MULTI-hop routing: No dedicated routers are necessary; every node acts as a router and forwards each other's packets to enable information sharing between mobile hosts.

5) MOBILITY: Each node is free to move about while communicating with other nodes. The topology of such an ad hoc network is dynamic in nature due to constant movement of the participating nodes, causing the intercommunication patterns among nodes to change continuously.

ADVANTAGES ARE:

1) ACCESSEBILITY: MANET provides access to information and services regardless of geographic position. 
2) DEPLOYMENT: The networks can be set up at any place and time.

3) INFRASTRUCTURE-LESS: The networks work without any pre-existing infrastructure. This allows people and devices to interwork in areas with no supporting infrastructure.

4) DYNAMIC: Can freely and dynamically self-organize into arbitrary and temporary network topologies.

\subsection{Design Issues and Challenges of MANET}

Ad hoc wireless networks inherit the traditional problem of wireless communication which includes the wireless channel unprotected from external signals, channel is not very reliable in certain circumstances, It is time varying and has asymmetric propagation properties, channel has no observable boundaries outside of which stations are known to be unable to receive network frames There are various problems and complexities, which includes their mobility, multi-hop nature, lack of fixed infrastructure, limited bandwidth, more power consumption add a number of characteristics, complexities, and design constraints that are specific to mobile ad hoc network:

1) Infrastructure-less networks: The most fundamental aspect of an ad hoc wireless network is its lack of infrastructure, and most designissues and challenges stem from this characteristic. Also, lack of ${ }^{\circ}$ centralized mechamism brings added difficulty in fault detection and correction.

2) Dynamic Topology: The dynamically changing nature of mobile nodes causes to the formation of an unpredicted lopology [4]. Thıs topology change causes frequent route change, network partitioning and packet dropping.

3) Limited Link Bandwidth and Quality: Because mobile nodes communicate each other via bandwidth-constrained, variable capacity, error-prone, and insecure wireless channels, wireless links will contihue to have significantly lower capacity than wired links, and hence-more problematic network congestion.

4) Power Constrained Operation: Power constraints are another big challenge in ad hoc wireless networkdesign [5]. These constraints in a wireless network arise due to battery powered nodes which cannot be recharged on line. This becomes a bigger issue in mobile ad hoc networks as each node is acting as both an end system and a router at the same time, and for the purpose, additional energy is required to forward packets.

5) Robustness and Reliability: Misbehaving nodes and unreliable links can have a severe Impact on overall network performance. Due to the lack of centralized monitoring [6] and management mechanisms, these types of misbehaviors cannot be detected and isolated quickly and easily. This increases the design complexity significantly.

6) Network Security: Mobile wireless networks are more vulnerable to information and physical security threats than fixed-wired networks [7]. Use of open and shared broadcast wireless channels results in nodes with inadequate physical protection that are prone to security threats. In addition, because a mobile ad hoc network is a distributed infrastructure-less network, it mainly relies on individual security solution from each mobile node, as centralized security control is hard to implement.

7) Quality of Service: Quality of Service (QoS) [8, 9, 10, 11, 12, 13, 14, 15, 16] guarantee is very much essential for the Successful communication of nodes in the network. As QoS provisioning is an important aspect for mobile ad hoc networks, similarly power conservation is a critical issue in ad-hoc wireless networks for node 
and network life, as nodes are battery powered only. Therefore, power consumption must also be treated as an indirect measure of QoS. The key factor is to maximize the time for network partition and reduces variations in power levels of nodes. The QoS metrics are throughput, packet loss, delay, jitter and error rate. It is hard to use these metrics directly in a network without any centralized control. It is very difficult in achieving the desired QOS guarantee because of the constraints in wireless channel.

8) Delay Tolerance: Mobile Ad Hoc Networks are autonomous and do not depend upon the fixed infrastructure that the network graph is rarely, if ever, connected. Most of the time DSR protocol fails to establish routes from source to destination because of lack of end-to-end connectivity between mobile nodes resulting in a lack of instantaneous end-to-end paths. So the main challenge of MANET is the disconnected nature between the mobile nodes. Hence the communication between the nodes must be delay-tolerant. Such networks are referred as Disconnected DelayTolerant MANETs (DDTMs). The challenges associated with mobile-computing are not new. However, issues in wireless communication such as low bandwidth, disconnections and high bandwidth variability are problematic and further exacerbated in DDTMs by little or no infastructure, variable node population and lossy links. Delay Tolerant MANETs additionally face chanlenges of mobility which is frequent and uncontrolled resulting in a highlyodyamic topology and disconnected network graph. In these challenging environments, popular ad hoc routing protocols such as AODV and DSR fail to establish routes. This occurs as a consequence of the fact that these protocols attempt to first establish a complete route and then, forward the actual data after the royte bals been established.

\section{Introduction to DSR Routing Protocol}

\subsection{DSR}

The Dynamic Source Routing protocol (DSR) [17] is a self maintaining protocol for wireless networks. Using DSR the network is completely self-organizing, self configuring which requires no fixed (infrastructure or centralization but is managed by human administrators. It is a roufing protocol for wireless mesh networks. It is similar to AODV in that it forms a route on-demand when a transmitting computer requests one. However, it uses source routing instead of relying on the routing table at each intermediate device. The protocol can also function with cellular telephone systems and mobile networks with up to about 200 nodes.

The address of each device between the source and destination is accumulated during route discover in order to determine source routes. The accumulated path information is cached by nodes processing the route discovery packets. The learned paths are used to route packets. To accomplish source routing, the routed packets contain the address of each device the packet will traverse. This may result in high overhead for long paths or large addresses, like IPv6. To avoid using source routing, DSR optionally defines a flow id option that allows packets to be forwarded on a hop-by-hop basis. There are two main components, called Route Discovery and Route Maintenance. Route Discovery determines the optimum path for a transmission between a given source and destination. Route Maintenance ensures that the transmission path remains optimum and loop-free as network conditions change, even if this requires changing the route during a transmission. 


\section{Power Aware Model}

1) Transmission Power- Whenever a node sends data packet to other nodes in the network, some amount of energy is required for transmission and such energy is called transmission Energy one important goal of a routing protocol is to keep the network functioning as long as possible during active and inactive communication energy. So if the mobile node power is minimized the goal of keeping the network alive can be accomplished. Transmission power control [18] and load distribution are two approaches to minimize the active communication energy, and sleep/power-down mode is used to minimize energy during inactivity. Two approaches to minimize the active communication energy $\left(T_{x}\right)$ of that node and this energy is dependent on size of the data packet. On sending the data packet, some amount of power is consumed. The transmission power is formulated as: $T_{x}=(330 *$ Plength $) / 2 * 10^{6}$ and $P_{t}=T_{x} / T_{t}$, Where $T_{x}$ is transmission energy, $R /$ is transmission power [19], $T_{t}$ is the time taken to transmit a data packet and Plength is the length of data packet in bits. Transmission power includes both power required to drive the circuit and the transmission power from antenna. The transmission power from antenna is computed based on the distance between sender and receiver using one of the models stated below.

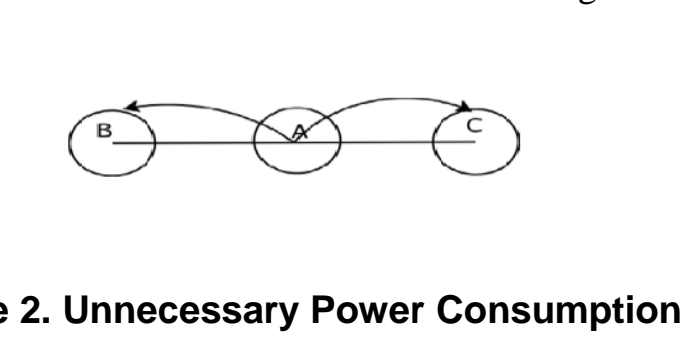

2) Reception Power- Whenever a node receives data packet from other nodes then some amount of energy is taken by the source node to receive data packet, which is called Reception (Power $[20]\left(R_{x}\right)$. On receiving the data packet some amount of power is consumed. Reception Power is formulated as: $R_{x}=(230 *$ Plength $) / 2 * 10^{6}$ and $P_{R}=R_{1} T_{r}$, Where $R_{x}$ is Reception Power, $T_{r}$ is the time taken to receive data packet and Plength is the length of data packet in bits.

Idle Power- In this situation, node neither transmits nor receives any data packets. Power is consumed because it needs to listen to the wireless medium continuously in order to detect a packet that it should receive, so that the node can then switch into receiving mode from idle mode. Idle power is a wasted power that should be eliminated or reduced to a minimum. Thus, Idle Power [21] is: $P_{I}=P_{R}$, Where $P_{I}$ is idle power and $P_{R}$ is reception power.

4) Overhearing Power- In this case a node picks up the data packets that are destined to other nodes and this is called overhearing and it may consume power. This power is called overhearing power. Unnecessarily receiving such data packets will cause 
power consumption. Then power consumed in overhearing is: $P_{\text {over }}=P_{R}$, Where $P_{\text {over }}$ is Overhearing Power [22] and $P_{R}$ is reception power.

\section{Power Efficient Dynamic Source Routing (PEDSR) for MANET}

\subsection{Introduction}

Without a fixed infrastructure, ad hoc networks have to rely on portable, limited power sources. What's more, a node in an ad hoc network has to relay messages for other nodes in the same network. The issue of power efficiency therefore becomes one of the most important problems in ad hoc networks. Power can be consumed during processing and communication. The power consumed during communication is more dominant than the power consumed during processing. So, the communication system should be power efficient or power aware by optimizing the power consumption at differen states of the communication. Power Aware Routing [23] is very essential in MANET.

\subsection{Motivation}

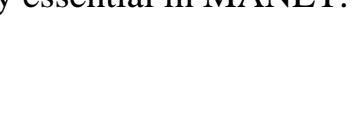

Power Aware routing technique significantlyaims at minimizing the power consumption of the network. We have gone through the literature survey of various MANET routing protocol. Each protocol has significant importance of its own as well as has some limitations. Based on the limitation constraint of nore power consumption in MANET we proposed a new power efficient dynamic soufce routing protocol which uses hop-by-hop power control mechanism to maximize the lifetime period of both node as well as network and reduces the total power consumption in the network.

\subsection{Proposed Method}

We have proposed a Power Efficient Dynamic Source Routing (PEDSR) which is based on Transmission power control approach. To reduce the transmission power we are using a hopby-hop power control mechanism [24]. Here during the route discovery phase itself we are calculating the minimum power required to communicate to the node which sends the request to it. The destination node will make a decision about the selection of best route among the multiple requests that reaches to it and sends reply packet to the destination through the selected route. We avoid the additional computations required to find out the route as well as the multiple Teplies to the source. The minimum power aware routing protocol is designed and implemented by making changes in the minimum-hop fixed-transmit power version of DSR. After adding power to the existing DSR protocol power gets minimized in the new proposed PEDSR protocol and the transmit power gets varied and no more fixed like existing DSR pyotocol. Minimum Power Routing protocol under Low Power Routing Protocol is used and the problem is stated as:

$$
\text { Minimize } \sum_{c \in \text { path }} P(c, c+1)
$$

Where $\mathrm{P}(\mathrm{c}, \mathrm{c}+1)$ denotes the power spent for transmitting and receiving between two consecutive nodes i.e. $\mathrm{c}$ and $\mathrm{c}+1$ (a.k.a link cost), in the route P. The link cost can be determined for both fixed as well as varied transmission power. 
PEDSR is derived from DSR protocol.PEDSR uses transmission power control approach which can be achieved with the help of topology control of a MANET. The range over which the signal is coherently received by the receiver is determined by the transmission power. PEDSR protocol based on transmission power finds the beat route that minimizes the total transmission power between a source and destination. Then comparisons are made between DSR and PEDSR protocol taking into considerations some of the important performance metrics which will be discussed later.

\subsection{Power Aware Dynamic Source Routing (PADSR) Protocol}

To obtain a power aware routing protocol we use power control approach i.e. Transmission power control approach. If we compare our proposed protocol PEDSR with previous works of researchers based on proposed model of DSR. In our proposed protoco PEDSR the idea behind is that, a hop-by-hop power control mechanism is ûsed to adjust the total power consumption of the network. Nodes rich in power are allowed to participate in routing process and over utilized nodes are avoided which may consume more power and thus in this way the life time of the network is improved. If there is a condition that the transmit power is fixed and equal for all the nodes, then minimum hop is the minimum power route. So in order to gain maximum power savings, the minimum power routing protocol should transmit the datapacket at power $P_{t}$ instead of the fixed transmit power. This can be achieved by applying dynamic transmit power control on the Tink. Now dynamic transmit power control is employed, the power cost of eách link can be computed using $P_{t}$ and other parameters. Though some protocols allow link metrics other than minimum hop, the existing on-demand protocols do not offer any mechanisms to compate and propagate the parameters necessary to compute the per packet powerdost. Hence the dynamic transmit power control feature cannot be supported by the existing versions of the on-demand protocols. Besides dynamic transmit power is used for power conservation, it is used to utilize the network resource efficiently. That is by allowing a greater number of simultaneous transmissions, the power control increase the total network capacity. In PEDSR, there is also an option that if the route tends to break earlier than the desired period that breakage is detected earlier and is avoided by adding Minimum- Power field. The remaining battery power of a mobile node is kept by the Minimum-Power fiefd When a node accepts a Route Request (RREQ) packet from its neighboring nodes it compares the Minimum-Power value in the packet with its remaining power. If the remaining power is less than Minimum-Power, this power is assigned as the Minimum-Poyer. This process will continue up to the destination. If more than one RREQ from differen route is accepted by the destination, the route having the highest value is selected in the Minimum-Power field and then Route Reply (RREP) packet is sent to the source. That means a route is selected by avoiding the node which is having the tendency to expire earlier. This way the route which may break early are eliminated [25]. A hop-by-hop control mechanism is used to save the remaining battery power in which the nodes that receives a Route-Request packet at power $P_{r}$ is transmitted with power $P_{t}$, then the new transmission power $P_{n}$ for this receiving node is calculated such that this node can Communicate with the sender node by using this minimum required power $P_{n}$ using the formula :

$$
P_{n}=P_{t}-P_{r}+P_{t h r}+P_{m}
$$


Where $P_{t h r}$ the required threshold which is considered as the power of the receiving node for successful reception of the packet and $P_{m}$ is the power included overcoming the problem of unstable and unreliable links due to wireless channel fluctuations. While sending back the Route-Reply packet it sends the same power to the sender node and it uses this power for data packet transmission. In a power table the calculated power at each node is stored and this is the minimum required power for successful transmission and reception. The node rebroadcasts the Route-Request packet with maximum power, if it is not the destination. The next hope node also does the same procedure and it will continue up to the destination. More than one RREQ packet may be available for the destination node from different available route from the source. It will select a route which is having more power in the Route-Request packet so that it can communicate with the destination node for a long time. The remaining request packet is simply ignored in the assumption that it cannot live for long time as compared to the selected packet. So the selected route does not have a node that may early die out and is power efficient [26]. Each pair of nodes in the route will ase the required transmission power for its successful communication. So through the selected route the destination node will send a Route-Reply packet to the source and the overhead of multiple Route-Reply packets are removed. So if we compare our proposed protocol PEDSR with existing DSR or the previous works of researchers based of proposed model on DSR first, our proposed protocol minimizes the consumption of power at both node and network level by using hop-by-hop power control mechanism., PEDSR protocol avoids usage of over utilized nodes and also reduces congestion of network.

\section{Simulation Result}

\subsection{Introduction}

We have done simulation work for our proposed PEDSR in Network Simulator (NS2) version ns-allinone-2.35. The simutation result shows that the proposed method PEDSR is better and efficient than the existing method i.e. DSR.

\subsection{Network Simulator 2}

NS-2 or Network Sinmulator [27] is a discrete-event simulator whose implementation was started by 1989 with the development of the Real Network Simulator. Earlier simulation of wired technology was done by NS-2, then the Monarch group from the Department of Computer Science at the University of Rice

developed the software for wireless mobile nodes. This contribution from the University of Rice is widely accepted all over the world. The main objective of NS-2 is to model the network protocols which includes wired network, wireless network, satellite, TCP, UDP, web, telnet, FTP, multicast, unicast, ad hoc routing and sensor networks. In NS-2 physical activities are translated to events. NS-2 [28] uses two languages C++ and Object Tool Command Language (OTCL) .C++ is fast to run but slower to change, making it suitable for detailed protocol implementation. OTCL runs much slower as compared to c++ but modification can be done very quickly (and interactively), making it ideal for simulation configuration. In NS-2, the front end of the program is written in TCL (Tool Command Language) and the backend of NS-2 simulator is written in C++ language. When the tcl program is compiled, two files that is trace file and nam file are created that defines the movement pattern of the nodes and also keeps track of the number of data packets sent by the source node, number of minimum hops between 2 mobile nodes, connection type at each instance of time etc. Moreover, a scenario file is created which defines the destination of 
mobile nodes along with their speeds and a connection pattern file (CBR file) or (TCP file) defining the pattern of communication, node topology and also the data packet type are also used to create the two files that is trace files and nam files which are then used by the simulator to simulate the network. NAM, the Network Animator is a Graphical User Interface and is used to visualize ns output and the trace file is used for post processing work. By using these trace files awk scripts can be written and using these awk scripts various performance metrics like Average Throughput, End to End Delay, Packet Loss, Packet Delivery Fraction, Packet Delivery Ratio, Normalised Overhead Routing etc can be calculated. Graphs are plotted using GNUPLOT in NS-2 which is a free, command-driven, interactive, function and data plotting program.

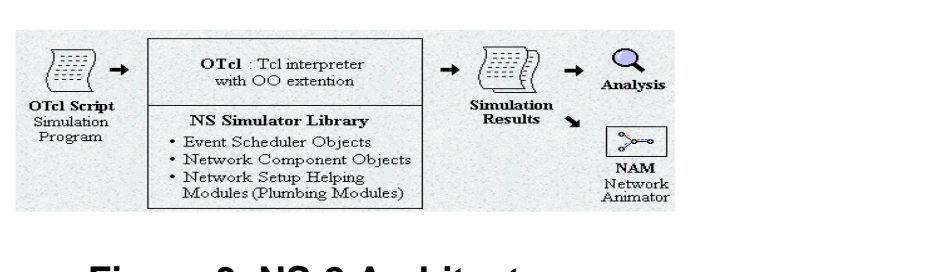

Figure 3. NS-2 Architecture

\subsection{Simulation Setup}

To evaluate the performance and power-efficiency of exísting routing protocol DSR, we used the event driven simulator ns-allinone-2.35 for our simulations. The parameters are chosen on three basic considerations.

1) Based on usage by other ressearchers, so that the results can be compared.

2) Based on the capabilities of the systems used for the simulation. For example, the simulation seems to rake much more tíme and memory when the number of nodes in the simulation is increased. So that limits the maximum number of nodes that can be used in this work.

3) By considering most of the results to show the effect of the energy challenges.

Hence, we try to reduce the dominancy of other factors, but we cannot avoid completely coping with realistic environmeht.

Table 1. General Parameters used in Simulation Having TCP traffic

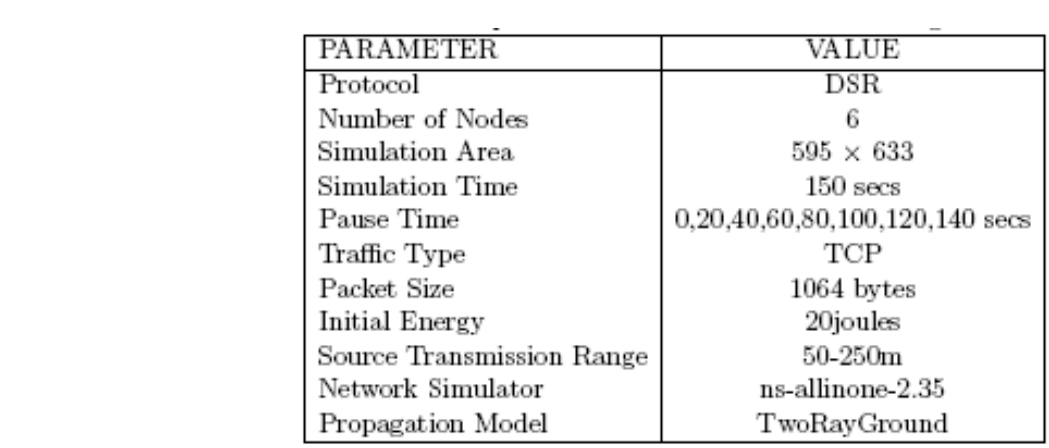

NS instructions provide a scalable simulation environment for wireless network systems and are used to define the topology of the network in a significant way. Traffic pattern type is taken as TCP and CBR [29] both. Table 5.1 describes the general parameters used in simulation having TCP traffic. The qualities of Transmission Control Protocol (TCP) traffic pattern are i) reliable: since connection is established prior to transmitting data, there is a guarantee that the data is being transmitted to the destination, ii) bi-directional: every packet 
that has to be transmitted by the source is acknowledged by the destination, and iii) conformity: there will be flow control of data to avoid overloading the destination and congestion control exists to shape the traffic such that it conforms to the available network capacity. Today more than traffic is carried out through TCP. Constant bit rate (CBR) means consistent bits rate in traffic are supplied to the network. The CBR service category is used for connections that transport traffic at a constant bit rate, where there is an inherent reliance on time synchronization between the traffic source and destination. CBR is tailored for any type of data for which the end-systems require predictable response time and a static amount of bandwidth continuously available for the life-time of the connection. In CBR, data packets are sent between source and destination with fixed size and interval. The receiving node does not send any acknowledgement messages on receiving the data packets. The medium access control (MAC) protocol is based on IEEE 802.11 distributed coordination fupction from CMU. Number of nodes are taken to be 6 with simulation time 150 seconds and Simulation Area to be $595 \times 633$. Propagation model is Two Ray Ground model and mobility model is Random Way Point Model .This model is the commonly used mobility model and destination is randomly chosen by every node and every node-moves towards the destination from a uniform distribution $\left(0, V_{\max }\right)$ at any moment of time, where $V_{\text {max }}$ is the maximum velocity allowable for every node. Each node stops for a time period defined by the pause time parameter when it reaches the destination. After the pause IIIne it again chooses a random destination and repeats the whole process until the end of the simulation. Table 5.2 describes the general parameters used in simulation having $\mathrm{CBR}$ traffic. An Omnidirectional antenna having unity gain is used by mebile nodes Interface Queue is implemented as a CMUPriQueue which gives pribrity to routing protocol packets by inserting them at the head of the queue. There are 2 scenarios one for TCP traffic having packet size to be 1064 bytes and other for CBR traffic having packet size 512 bytes. Considering these 2 traffic patterns DSR [30] is compared with new proposed protocol PEDSR with respect to performance metrics: Average Throughput, End to End Delay, Normalised Routing Overhead, Packet Delivery Fraction, Packet Delivery Ratio and Number of Dropped Packets which are discussed in the next section.

Table 2. General Parameters used in Simulation Having CBR traffic

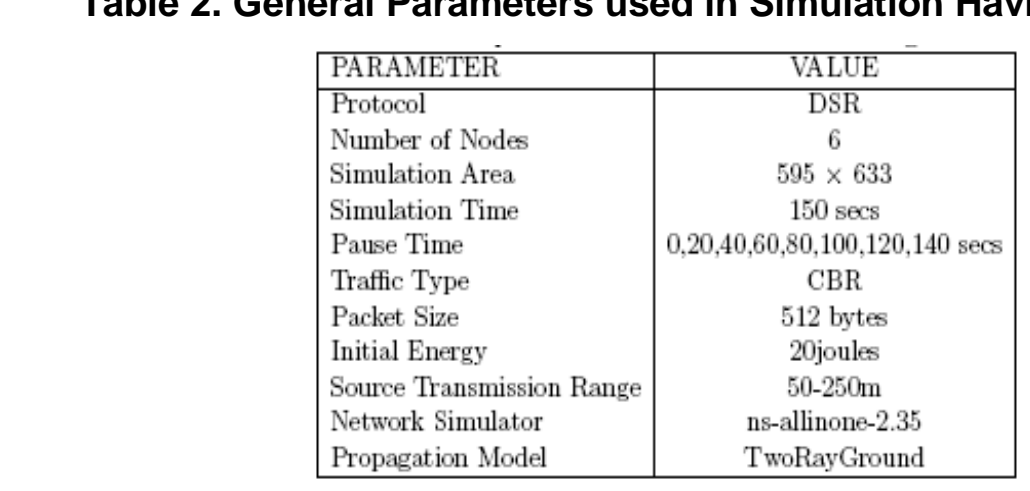

\subsection{Performance Evaluation Metrics}

5.4.1. End to End Delay: This metric is defined as the time taken by a packet to be transmitted across a network from source to destination node. It is measured in seconds. With the traffic pattern TCP having packet size 1064 bytes, the graph in figure 4 shows the performance evaluation of DSR and PEDSR with respect to this end to end delay metric. The graph shows that PEDSR performs better than DSR in both the traffic type i.e. CBR and TCP. 
For Traffic type TCP in high mobility (0 pause time) delay in DSR is $131 \mathrm{~s}$ and as the pause time increases delay is constant but in case of PEDSR delay in high mobility scenario is $127 \mathrm{~s}$ which is less than DSR, then as pause time increase delay goes on decreasing for every 20s. In low mobility scenario delay is very less in PEDSR which is 79 seconds. So in terms of End to End Delay with varied pause time DSR is uniform but in PEDSR delay goes on decreasing and is less as compared to DSR. So it shows that PEDSR outperforms DSR and gives better result. With the traffic pattern CBR having packet size 512 bytes, the graph in figure 5 shows that PEDSR outperforms DSR. In high mobility scenario end to end delay in DSR is $123 \mathrm{~s}$ and with varied pause time the delay is uniform. In case of PEDSR the end to end delay is $123 \mathrm{~s}$ in high mobility scenario and as time increases the delay goes on decreasing. In low mobility area the delay is very less so it shows that our proposed protocol PEDSR is better than DSR.

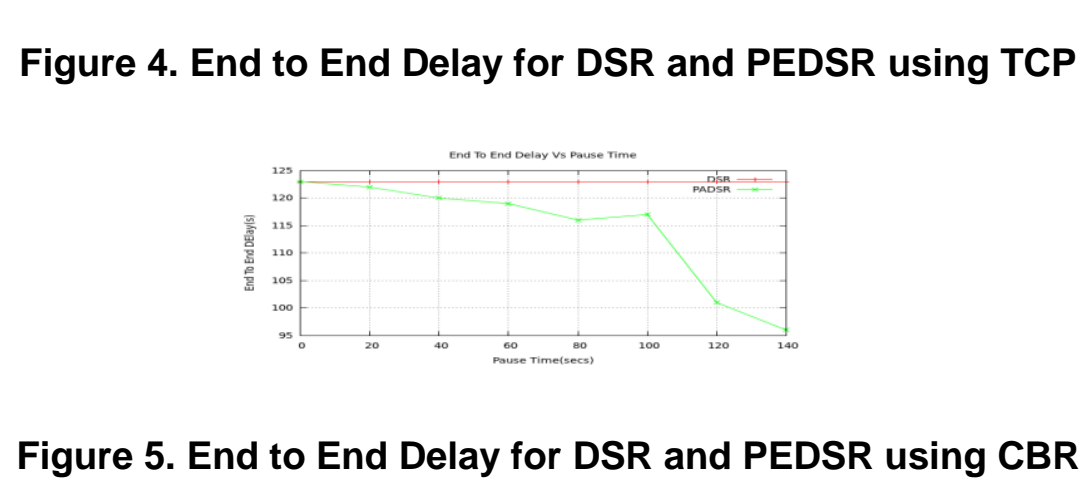

5.4.2. Average throughput: Throughput metric represents the total number of bits forwarded to higher layers persecond. It is measured in Kbps. It can also be defined as the total amount of data a receiver actually receives from sender divided by the time taken by the receiver to obtain the last packet. It is the average rate of successful message delivery over a communication channel.

Formula for Average Throughput is:

(Received Size/ (stop Time - start Time)) * (8/1000).

In terms of Average Throughput with varied pause time and with TCP traffic PEDSR performs same as DSR. In high mobility area (Zero pause time) throughput is $620 \mathrm{kbps}$ in DSR and with varied pause time throughput is uniform. In PEDSR throughput is $485 \mathrm{kbps}$ in high mobility area and with varied pause time it gradually increases. The graph in figure 6 shows that at 120 seconds the average throughput is $620 \mathrm{kbps}$ and is uniform in 140 seconds that is $620 \mathrm{kbps}$ in low mobility area. So it shows that PEDSR performs better in low mobility area. In terms of Average Throughput with varied pause time and with CBR traffic PEDSR performance is low as compared to DSR protocol. The graph in figure 7 shows that in high mobility area (Zero Pause time) throughput is $583 \mathrm{kbps}$ but with varied pause time throughput increases and in low mobility area that is at 140s the average throughput is $605 \mathrm{Kbps}$ and is 
lower as compared to DSR whose average throughput is $608 \mathrm{Kbps}$ and is uniform with varied pause time.

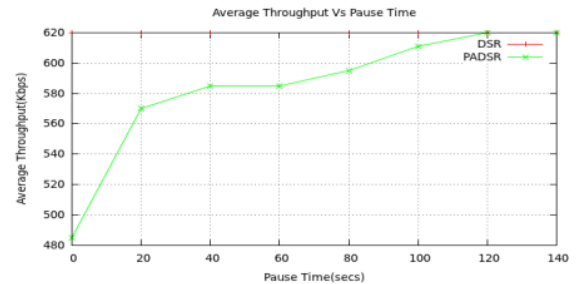

Figure 6. Average Throughput for DSR and PEDSR using TCP

Figure 7: Average Throughput forDSRand PEDSR using CBR

5.4.3. Normalized Routing Overhead: This metric is defined as the total number of routing packets transmitted during simulation. In terms of normalized routing overhead with varied pause time and with TCP traffic type the graph in figure 8 shows that the normalized routing overhead in DSR is 0.014 and is uniform both high mobility and low mobility area. But in case of PEDSR in high mobility area i.e. at Zero pause time normalized routing overhead is 0.118 which is vers high as compared to DSR and as pause time increases at 20s overhead increases and is is 0.147 then at $40 \mathrm{~s}$ again decreases, at $60 \mathrm{~s}$ increases and at $80 \mathrm{~s}$ overhead increases at a very high rate and is 0.205 and then decreases gradually in low mobility area and at 140s overhead is 094 . In terms of normalized routing overhead with varied pause time and with CBR traffictype the graph in figure 9 shows that the overhead in DSR is 0.004 and is uniform in both hígh and low mobile area. In PEDSR the overhead in high mobile area is 0.389 which is high as compared to DSR protocol and as pause time increases overhead also varies i.e. at $20 \mathrm{~s}$ overhead is 0.528 and then it decreases at $40 \mathrm{~s}$ and is 0.212 , at $60 \mathrm{~s}$ overhead is low and is 0.020 then suddenly overhead increases at $80 \mathrm{~s}$ and is 0.500 and then overhead gradually decreases in low mobile area and is very low at 140 s and is 0.018 .

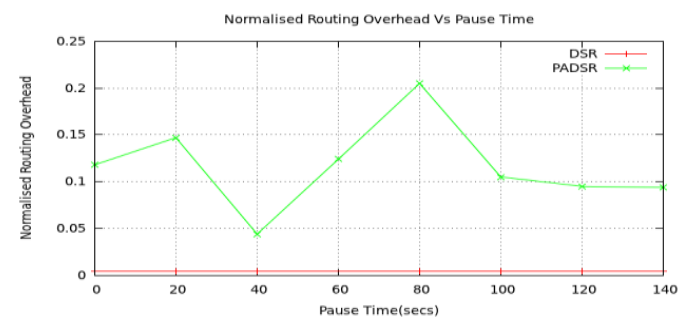

Figure 8. Normalized Routing Overhead for DSR and PEDSR using TCP 


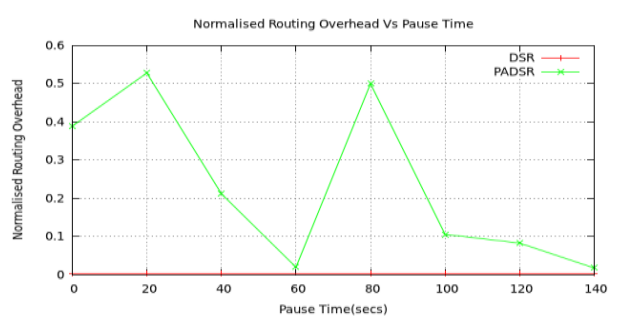

Figure 9. Normalized Routing Overhead for DSR and PEDSR using CBR •

5.4.4. Number of dropped packets: This metric is defined as the measure of the number of routing packets dropped by the routers due to various reasons. The possible reasons for packet dropping we have considered $\mathrm{f}$ is Collisions, time outs, looping, enors etc. In terms of number of dropped packets with TCP traffic type number of dropped packets in PEDSR is less as compared to DSR protocol in high mobility area dropped packets are 103 and varies as pause time increases. The graph in figure 10 shows that at 20 and 40 s dropped packets are uniform and is 200. At 60s dropped packets decreases and then at 80 s increases and then decreases at 100s, and then in low mobility area number of dropped packets increases. In PEDSR in high mobility area number of dropped packets are less and is 99 and as pause time increases dropped packets varies. The graph in figure shows that at 20 and $40 \mathrm{~s}$ dropped packets are uniform then at 60s dropped packets increases and then decreases, then at 100 and $120 \mathrm{~s}$ dropped packets are uniform and in low mobility area $i . e$. at $140 \mathrm{~s}$ the number of dropped packets increases. PEDSR performs better than DSR. In terms of number of dropped packets with CBR traffic type number of dropped packets is less in PEDSR as compared to DSR. The graph in figure 11 shows that in high mobility area number of dropped packets are less and as pause time increases dropped packets varies. At pause time 20 s the number of dropped packets are less and then increases and at 60s again decreases, at 80s the graph increases then decreases and then in low mobility area increases. In PEDSR in low mobility area number of dropped packets are less and with pause time varies. At pause time 20s, 40s and 60s number of dropped packets increases, at 80s and 100s the graph decreases and then increases up to 140s. S6in low mobility area number of dropped packets in PEDSR increases but is less than DSR Soyt shows number PEDSR outperforms DSR.
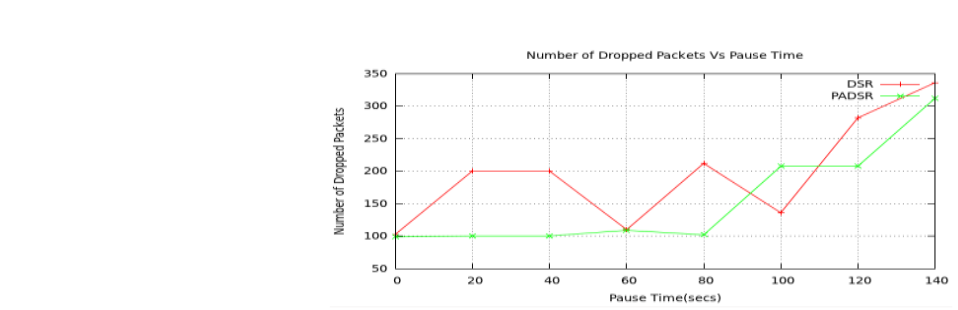

Figure 10. Number of dropped Packets for DSR and PEDSR using TCP 


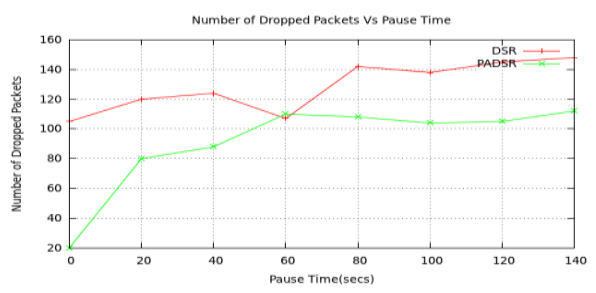

\section{Figure 11. Number of dropped Packets for DSR and PEDSR using CBR}

5.4.5 Packet delivery ratio: Packet delivery ratio is the ratio of number of packets reeeived at the destination to the number of packets sent from the source. In terms of Racket Delivery Ratio with TCP traffic type in DSR the graph in Figure 12 shows that in high mobility area the packet delivery ratio is 0.9725 and is constant with varied pause time. But in PEDSR in high mobility area PDR is 0.9921 and is high and as pause time increases packet delivery ratio varies. But with CBR traffic type in DSR the graph in figure 13) shows that in high mobility area i.e. at 20s packet delivery ratio is 0.9942 and then goes on decreasing up to 140s i.e. in low mobility area. So PEDSR outperforms DSR in terms of packet delivery ratio with both the traffic type.

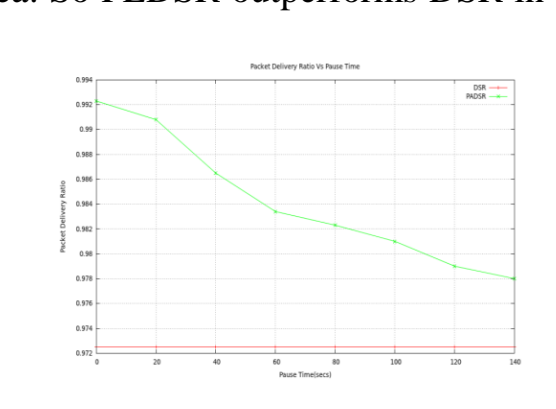

Figure 12. Packet Delivery Ratio for DSR and PEDSR using TCP

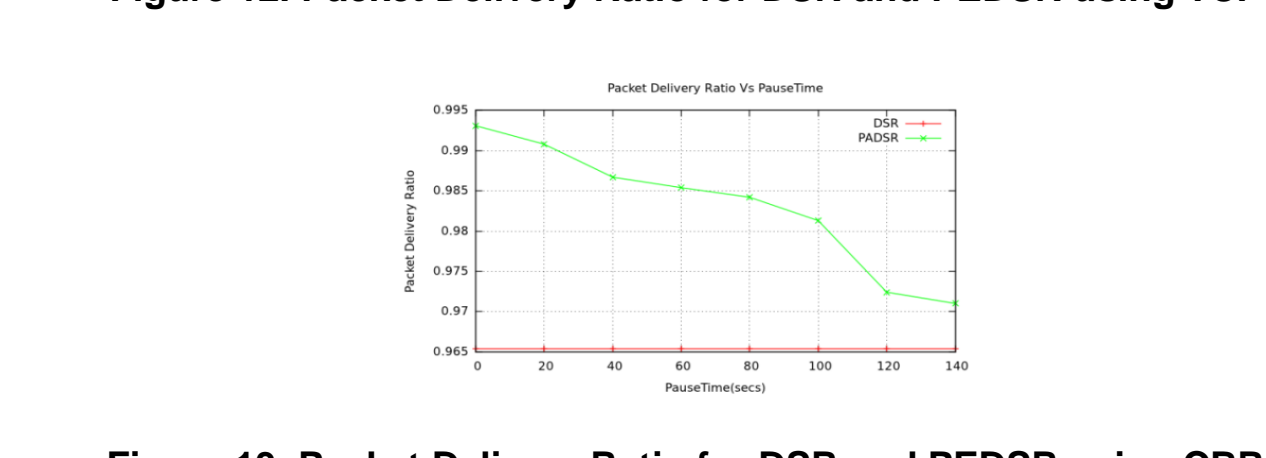

Figure 13. Packet Delivery Ratio for DSR and PEDSR using CBR

\section{Comparisons}

If we compare our proposed protocol (PEDSR) Power Efficient Dynamic Source Routing protocol with other power aware routing protocols whose detailed study had been carried out by many researchers then many conclusions can be drawn out regarding which protocol is better in saving the battery life time of a mobile node based on different mechanisms and algorithms which will be helpful for the future point of view. One such protocol is MER [31] (Minimum Energy Routing) protocol which uses the transmission power control approach and uses the power information such as node and link cost as compared to our proposed 
protocol PEDSR which uses hop-by-hop mechanism in order to calculate the minimum power required for the successful transmission from the sender to destination. Limitation of MER protocol is that there is a chance that node may fail because more flows are introduced by minimum energy routes and in these routes nodes exhausts their energy at early stage hence the network is incapable of performing any task due to the failure of the nodes but this is not the case in PEDSR protocol, nodes retain their energy till the data packets are sent from source to destination.

If we compare PAAODV [32] (Power Aware Ad Hoc On-demand Distance Vector Routing) Protocol with PEDSR protocol then we find that PAAODV protocol optimally reduces the power transmission to a minimum power level without hampering the connectivity of the network. In the Route discovery process a source node initially discovers a path with a low power level. If it is unable to find a path with this power level, then it attempts further with a higher power level. Using two different power levels in the route discovery phase reduces route discovery time and at the same time reduces the overhead too as compared to that in MER but in case of PEDSR protocol the remaining battery power of a mobile node is kept by the Minimum-Power field. When a node accepts a Route Request (RREQ) packet from its neighboring nodes it compares the Mininum-Power value in the packet with its remaining power. If the remaining power is less than Minimum-Power, this power is assigned as the Minimum-Power, This process wilfecontinue up to the destination so congestion of the network is reduced as wetl as the overhead is also low if we compare with previous two protocols.

Another such protocol is COMPOW [33] protocol which guarantees bi-directionality of links. The main advantage of this protocol is that it provides power aware routes, increases the traffic carrying capacity, reduces the battefyconsumption, latency and interference. But if we compare COMPOW protocol with PEDSR protocol then this protocol works only for homogeneous networks. When the nodes in a network are grouped, COMPOW protocol leads to high power level which is not in the case of PEDSR protocol as it works for heterogeneous networks and leads to ninimum power level by topology control approach.

If we compare PLR [34] (Power-aware Localized Routing) protocol with PEDSR protocol then we find that in PLR protocol a source node has the location information of its neighboring as well as destination node that means when a source node sends the data packet to destination node either they send the packets directly or through the help of neighboring nodes. This protocol implements a localized, fully distributed power aware routing algorithm. Advantage of PLR protocol is that the source cannot find the optimal path but selects the next hop through which the overall transmission power to the destination is minimized but one of the limitations is that if there is a direct transmission of data packets then more power is consumed which causes link error that would result in more retransmissions as compared to indirect transmissions through neighboring nodes but in PEDSR protocol even if it is direct or indirect transmission power is minimized because a route is always selected by avoiding the node which is having the tendency to die out earlier so there is no chance of link errors or any possibility of packet retransmission.

There is a protocol called as OMM (Online Max-Min) [35] protocol if we compare this protocol with PEDSR protocol then we find that OMM protocol finds the optimum path using Dijkstra algorithm and without requiring the information regarding the data transmission sequence or data generation rate the protocol makes a routing decision that optimizes the two different metrics that is minimizing power consumption (min-power) and maximizing the minimal residual power (max-min) but in case of PEDSR there is also an option that if the route tends to break earlier than the desired period that breakage is detected earlier and is avoided by adding Minimum- Power field. The remaining battery power of a mobile node is 
kept by the Minimum-Power field. When a node accepts a Route Request (RREQ) packet from its neighboring nodes it compares the Minimum-Power value in the packet with its remaining power. If the remaining power is less than Minimum-Power, this power is assigned as the Minimum-Power. This process will continue up to the destination. If more than one RREQ from different route is accepted by the destination, the route having the highest value is selected in the Minimum-Power field and then Route Reply (RREP) packet is sent to the source. In PEDSR protocol data transmission sequence or data generation rate is usually known in advance as compared to OMM protocol and also in this case each node is provided with only the local information which is not so in case of PEDSR protocol.

\section{Related work}

Like PEDSR protocol many power aware routing protocols have been implemented by various researchers earlier. PAOD [36] is a power-aware on-demand routing protocol whose main objective is to maximize the network lifetime of Mobile Ad Hoc Network. Here an important component that is power threshold is introduced that allows the mobile nodes to participate in the routing path but the required power may deplete before the session completes so there is no guarantee.

PAMP [37] (Power-Aware Multi-Path Routing Protocôl forg Wireless Ad hoc Network) is a power aware multipath routing protocol. The main objective of this protocol is increasing the availability of wireless as well as giving guarante of reliable and effective delivery of packets by exploiting low power nodes, It is specifically designed as an extension of AODV protocol. This protocol supports power reservation and multiple paths.

LAMOR [38] (Lifetime-Aware Multipath Optimized Routing Algorithm for Video Transmission over AdoHoc Networks) (s another power aware Multi-Path Routing Protocol whose objective is to extend the life time of the nodes, networks and path. It supports high speed real time video transmissipn oyer wireless ad hoc networks. It proposes a distributed rate allocation algo ithm based on the discovered multiple paths which extends the lifetime of the networks andimproves the yideo quality.

Power Aware Dynamic Source Routing Protocol to Increase Lifetime of Mobile Ad Hoc Networks (PADSR and Survival DSR) [39] is a novel power aware multipath routing protocol. In this protocol the routing algorithm is changed between the mobile nodes which establishes multi hop path and among all possible paths the node having high level of power is chosen. Secondly the algorithm is modified when the power of a node that is forwarding data within multi hop path reaches a level less than or equal to a certain threshold percentage of initial power. To avoid consumption of residual power the node looks for another path with the help of its neighboring nodes.

PDTORA [40](Power and Delay aware Temporally Ordered Routing Algorithm) protocol is an extension of TORA protocol with power and delay aware modification. With the help of a query packet at each node along the path between source and destination verification of power and delay requirements is carried out. This protocol maintains the topology information involving its one-hop neighbors. During query phase the nodes in the network which do not satisfy the QOS requirement of maximum delay and minimum power levels are eliminated from the route during communication. 
PAMAS [41] (Power Aware Multi-access) protocol saves power by turning off the radio signals. It uses a new routing cost model to avoid the use of nodes running low on battery power. This power aware protocol works only in the routing layer and exploits only routingspecific information. The PAMAS protocol saves 40-80 percentage of battery power by intelligently turning off radios when they cannot transmit or cannot receive data packets. . The lifetime of the network is improved significantly.

PDTMRP [42] (Power-aware dual-tree-based multicast routing protocol for mobile ad hoc networks) protocol is derived from MAODV (Multicast ad hoc on-demand distance vector routing protocol). This power-aware protocol improves the route stability of multicast routing. Moreover, this protocol is called as dual- tree based because it achieves the load balance of data transmission. Firstly, the protocol divides the multicast data packets into two parts and sends each part over a different tree. Secondly, the dual trees for data transmission were constructed by grouping the nodes. The main objective of the protocol is to maximize the network life time by load balancing approach and also the control overhead for route construction and the number of route reconstructions can be minimized.

PARO [43] (Power-aware Routing Optimization) protocol minimizes the transmission power consumed in the network. The princrpar behind this protocol is that one or more intermediate nodes called "redirectors" chooses to forward data packets on behalf of sourcedestination pairs and thus reduces the aggegate transmission power consumed by wireless devices. PARO uses redirector podes to shorten the length of individual hops, thereby reducing the overall power consumption.

\section{Conclusion}

We have simulated and eompared two protocols DSR and our proposed protocol PEDSR in different simulation scenarios and observing their behavior in terms of six significant parameters i.e, End To End Delay, Average throughput, Normalized Routing Overhead, Number of Dropped Packets and Packet Delivery Ratio in order to find out which one should be preferred when the mobile ad hoc network has to be set up for the particular duration. The whole simulation scenario consisting of maximum 6 nodes which are created by writing the OTCL script in NS-2 (yersion 2.35) and analyzing the parameters with the help of Gnuplot which is a data plotting program.

We have simulated DSR protocol considering both the traffic types i.e. TCP and CBR, then we added energy model to the DSR protocol with initial energy $20 \mathrm{j}$, transmission power 0.9 , receiving power 0.8 , idle power 0.0 and sense power 0.0175 and we got our proposed protoeol PEDSR. We simulated PEDSR taking two scenarios into consideration, in $1^{\text {st }}$ scenario we considered the traffic type to be TCP and in

$2^{\text {nd }}$ scenario we considered the traffic type to be CBR. By studying and analyzing the outputs appeared in GNUPLOT we come to this conclusion that PEDSR protocol must be preferred over DSR protocol in most of the scenarios. In terms of End to end delay PEDSR performs better than DSR as delay is less as compared to the DSR protocol in both the traffic type TCP and CBR. In traffic type CBR in terms of End to end delay PEDSR outperforms DSR protocol. In terms of average throughput in traffic type TCP PEDSR performs better in low mobility area and is uniform as compared to traffic type CBR. So with traffic type TCP in terms of average throughput PEDSR outperforms DSR protocol. In terms of normalized routing overhead with both the traffic type CBR and TCP in PEDSR overhead decreases in low mobility area as compared to high mobility area. Overhead in PEDSR is more as 
compared to DSR protocol. In terms of number of dropped packets with traffic type TCP PEDSR performs better than DSR. In low mobility area number of dropped packets in PADSR is low as compared to DSR. But with traffic type CBR PEDSR performs much better as compared to the traffic type TCP as the number of dropped packets is very less as compared to DSR protocol. So in both the traffic type PEDSR outperforms DSR protocol. In terms of packet delivery ratio PEDSR outperforms DSR with traffic type TCP. In high mobility area packet delivery ratio is high but as pause time increases packet delivery ratio varies and in high mobility area packet delivery ratio in PEDSR is high as compared to DSR whose packet delivery ratio is low and is uniform. Now with traffic type CBR PEDSR performance is also good. So we conclude that in most of the scenarios our proposed protocol PEDSR outperforms DSR protocol.

\section{Future Work}

The work can be further extended by implementing the scenario with the different mobility models and different network scenarios. Also the behavior of the protocols can be studied further by carrying the simulations on different parameters like varying the number of mobile nodes, the topology area choice of the traffic type between the mobile nodes other than the simulation time. The continuity of this work could be accomplished through the evaluation of others routing protocols (secure and not secure) particularily multipath routing protocols. Another interesting work that could be developed is to analyze the acting of security routing protocols in an Ad hoc network composed by malicious nodes.

\section{References}

[1] S. Nagaprasad, /(IJCSE) Interpational Journal On Computer Science and Engineering, vol. 02, no. 08, (2010), pp. 745-2748.

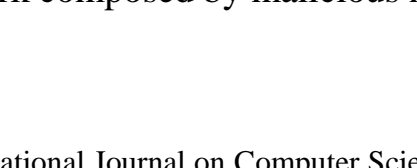$$
\text { pp. } 745-2748 \text {. }
$$

[2] S. M. Chen, P. Lin, D. Whang and S 4 R Yang, "A study on distributed/centralized scheduling for wireless mesh network", in Proceedings of the 2006 International Conference on Wireless Communications and Mobile Computing, Vancouver, British Columbia, Canada, (2006), pp. 599 - 604.

[3] H. Zhang, A. Arora, Y. Choi and M. G. Gouda, "Reliable Bursty Converge cast in Wireless Sensor Networks", Proceedings of the 6th ACM International Symposium on Mobile Ad Hoc Networking and Computing (MobiHoc05), Urbana- Champaign, IL, (2005) May.

[4] B. K. Pattanayak, M. K. Mishra, A. K. Jagadev and A. K. Nayak, "Power Aware Ad Hoc On- demand Distance Vector (PAAODV)", Routing for MANETS Journal of Convergence Information Technology, vol. 6, no. 6, (2011) June.

[5] S. Mishra and B. K. Pattanayak, "Power Aware Routing in Mobile Ad Hoc Networks: A Survey", ARPN Journal of Engineering and Applied Sciences, vol. 8, no. 3, (2013) March.

[6] S. R. Biradar H. H. D. Sharma, K. Shrama and S. K. Sarkar, "Performance Comparison of Reactive Routing Protocors of MANETs using Group Mobility Model”, IEEE International Conference on Signal Processing Systems, (2009), pp. 192-195.

[7] W Almobaideen, K. Hushaidan, A. Sleit and M. Qatawneh, "A Cluster based Approach for Supporting QoS in Mobile Ad Hoc Networks", International Journal of Digital Content Technology and its Applications, vol. 5, no. 1, (2011) January.

[8] W. Almobaideen, K. Hushaidan, A. Sleit and M. Qatawneh, "A Cluster based Approach for Supporting QoS in Mobile Ad Hoc Networks", International Journal of Digital Content Technology and its Applications, vol. 5, no. 1, (2011) January.

[9] L. Chen and W. B. Heinzelman, "A Survey of Routing Protocols that Support QoS in Mobile Ad Hoc Networks", IEEE Comm. Surveys and Tutorials, vol. 21, no. 6, (2007), December, pp. 30-38.

[10] L. Chen and W. Heinzelman, "QoS-aware Routing Based on Bandwidth Estimation for Mobile Ad Hoc Networks", IEEE JSAC, Special Issue on Wireless Ad Hoc Networks, vol. 23, no. 3, (2005) March, pp. 56172.

[11] P. Van Mieghem and F. A. Kuipers, "Concepts of exact quality of service algorithms", IEEE/ACM Transaction on Networking, vol. 12, no. 5, (2004), pp. 851-864. 
[12] T. Bheemarjuna Reddy, "Quality of Service Provisioning in Ad Hoc Wireless Networks: A Survey of Issues and Solutions", Ad Hoc Networks, vol. 4, no. 1, (2006) January, pp. 83-124.

[13] X. Masip-Bruin and M. Yannuzzi, "Research challenges in QoS routing", Computer Communications, vol. 29, (2006), pp. 563-581.

[14] B. K. Pattanayak, A. K. Jagadev, M. K. Mishra and M. R. Nayak, "A Distributed Cluster Scheme for Bandwidth Management in Multi-hop MANETs", (IJCSNS) International Journal of Computer Science and Network Security, vol. 9, no. 10, (2009) October.

[15] V. Rishiwal, S. Verma and S. K. Bajpai, "QoS Based Power Aware Routing in MANETs", International Journal of Computer Theory and Engineering, vol. 1, no. 1, (2009) April, pp. 1793-8201.

[16] P. Deepalakshmi and S. Radhakrishnan, "Ant Colony Based QoS Routing Algorithm For Mobile Ad Hoc Networks, International Journal of Recent Trends in Engineering, vol. 1, no. 1, (2009) May.

[17] SreeRangaRaju and J. Mungara, "Performance Evaluation of ZRP over AODV and DSR in Mobile Adhoc Networks using Qualnet”, European Journal of Scientific Research, vol. 45, (2010), pp. 658-674.

[18] G. Allard, P. Minet, D. Q. Nguyen, and N. Shresta, "Evaluation of the energy consumptiop in MANET, Adhoc-Now 2006, Ottawa, Canada, (2006) August.

[19] V. Kawadia and P. R. Kumar, "Principles and Protocols for Power Gontrol in Ad Hoc Networks", JSAC: Special Issue on Ad Hoc Networks, vol. 1, (2005) January.

[20] S. Buruhanudeen, M. Othman, M. Othman, and M. Ali, "Existing MANET Routing Protocols and Metrics used Towards the Efficiency and Reliability-An Overview, Proceedings of the 2007 IEEE international conference on Telecommunications and Malaysia Internationa Conference) on Communications, ICTMIXCC, Penang, Malaysia, (2007), pp. 231-236.

[21] P. Latha and R. Ramachandra, "Energy reduction aware multicast routing for mobile ad hoc networks", Proc. of Indian, Journal of Science and Technology, vol 3, no. 3, (2010) March pp. 305-307.

[22] R. Patil and A. Damodaram, "Cost Based Power Aware Cross Layer Routing Protocol For Manet", IJCSNS International Journal of Computer Science and Network Security, vol. 8, no. 12, (2008) December.

[23] Y. Yoo and D. P. Agrawal, "Optimal transmission power with delay constraints in 2D and 3D MANETs", Journal of Parallel and Distributed Compating, vol. 71, ro. 11,(2011) November, pp. 1484-1496.

[24] A. Samanta and D. Bakshi, "Faul Tolerant Wireless/Sensor MAC Protocol For Efficient Collision Avoidance", International journal on applications of graph theory in wireless ad hoc networks and sensor networks (GRAPHHOC), vol 2 , no. 2, (2010) June.

[25] V. J. Shine and S. K. Rath An Energy Eificient Dynamic Source Routing Protocol", In proceedings of National Conference on Recent Trends in Innoyative Technologies, (2009), pp. 8-10.

[26] M. Tarique and R. Islan, "Minimum Energy Dynamic Source Routing Protocol for Mobile Ad Hoc Networks", Internationar Journal of Computer Science and Network Security, vol. 7, no. 11, (2007) November.

[27] M. Hasanlou "Introduction 6 Mobility and Network Simulator 2 (NS- 2)", Department of Computer Engineering Sharif University of Technology.

[28] The VINT Project, The ns Manual, December 2003, http://www.isi.edu/nsnam/ns/ ns-documentation.html.

[29] B. P. Md. Ibrahim and Md. A. N. Bikas, "Performance Evaluation of Aodv and DSR with Varying Pause Time and Node Density Over TCP and CBR Connections in Vanet", IJCSNS International Journal of Computer Science and Network Security, vol. 11, no. 7, (2011) July.

[30] V. J. Shine and S. K. Rath, "A Novel Approach for Energy Efficiency in MANET", in Proceedings NCEIT09, (2009) 21 March.

[31] G.Jayakymar and G. Ganapathy, "Performance Comparison of Mobile Ad-hoc Network Routing Protocol", IJCSNS International Journal of Computer Science and Network Security, vol. 7, no. 11, (2007) November .

[32] B. K. Pattanayak, M. K. Mishra, A. K. Jagadev and A. K. Nayak, "Power Aware Ad Hoc On-demand Distance Vector (PAAODV) Routing for MANETS Journal of Convergence Information Technology", vol. 6, no. 6, (2011) June.

[33] Y. Yoo and D. P. Agrawal, "Optimal transmission power with delay constraints in 2D and 3D MANETs", Journal of Parallel and Distributed Computing, vol. 71, no. 11, (2011) November, pp. 1484-1496.

[34] J. Kao and R. Marculescu, "Predictive Energy-Efficient Multicast for Large Scale Mobile Ad-Hoc Networks", Proceedings of the Consumer Communications and Networking Conference, (2008) January, pp. 709-713.

[35] A. Samanta and D. Bakshi, "Fault Tolerant Wireless Sensor MAC Protocol For Efficient Collision Avoidance", International journal on applications of graph theory in wireless ad hoc networks and sensor networks (GRAPH-HOC), vol. 2, no. 2, (2010) June.

[36] W. Kun, "Power-aware on-demand routing protocol for MANET", _ in Proc. of ICDCSW, pp. 723-728, (2004) March. 
[37] J. S. Yang, K. Kang, Y.-J. Cho and S. Y. Chae, "PAMP: Power-Aware Multi-Path Routing Protocol for a Wireless Ad hoc Network", Wireless Communications and Networking Conference, 2008, WCNC 2008, IEEE, pp. 2247 - 2252, (2008) April.

[38] L. Tan, "LAMOR: Lifetime-Aware Multipath Optimized Routing Algorithm for Video Transmission over Ad Hoc Networks", in Proc. of IEEE VTC 2006-Spring, (2006), pp. 623-627.

[39] Md S. Akhter and V. P. Singh, "Power Aware Dynamic Source Routing Protocol to Increase Lifetime of Mobile Ad Hoc Networks", International Journal of Innovative Research and Development, vol. 2, no. 6, (2013) June.

[40] A. K. Jagadev, B. K. Pattanayak, M. K. Mishra and M. Nayak, "Power and Delay Aware On- Demand Routing For Ad Hoc Networks", (IJCSE) International Journal on Computer Science and Engineering, vol. 02, no. 04, (2010), pp. 917-923.

[41] D. Sharma and A. Kush, "Power and Mobility Aware Protocol for Ad Hoc Network," IACSIT International Journal of Engineering and Technology, vol. 3, no. 2, (2011) April.

[42] W. Wei and A. Zakhor, "Multiple Tree Video Multicast Over Wireless Ad Hoc Networks," IEEE Transactions on Circuits and Systems for Video Technology, (2007) January, pp. 2-15.

[43] J. Gomez-Castellanos, A. T. Campbell, M. Naghshineh, and C. Bisdikian, "PARO: Supporting transmission power control for routing in wireless ad hoc networks", Journal on Wireless Networks) (WINET), vol. 9, no. 5, (2003), pp. 443-460.

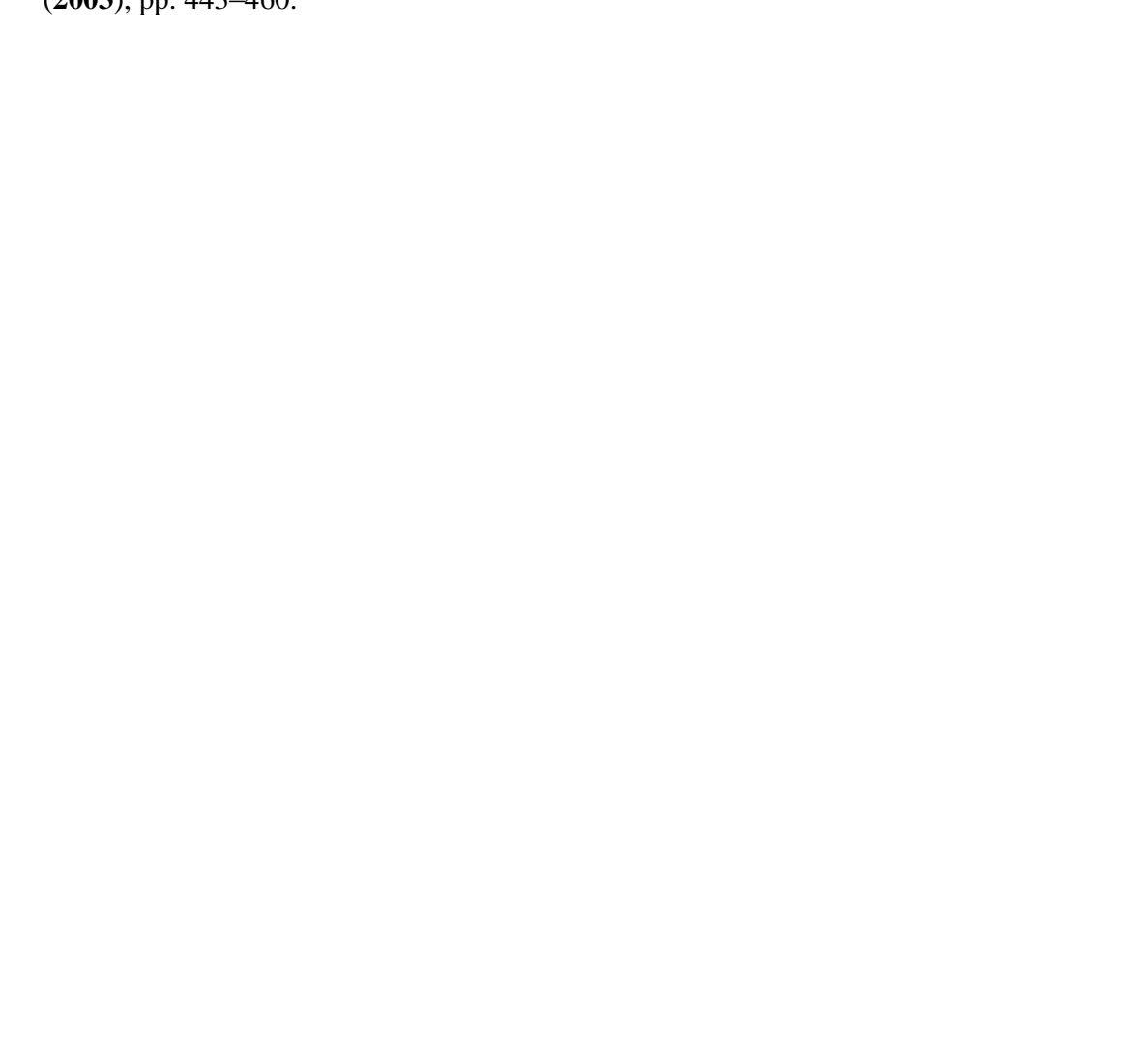

